\title{
A Hybrid Modular Multilevel Converter with Reduced Full-Bridge Submodules
}

\author{
Rui Li, Lie Xu, Senior Member, IEEE, Lujie Yu, and Liangzhong Yao, Senior Member, IEEE
}

\begin{abstract}
A hybrid modular multilevel converter (MMC) with reduced full-bridge $(\mathrm{FB})$ submodules $(\mathrm{SMs})$ is proposed, where a high voltage rating half-bridge $(\mathrm{HB})$ based $\mathrm{MMC}$ is connected in series with a low voltage rating FB-MMC in parallel with a fault breaking circuit on its DC side. Unlike conventional hybrid MMCs with mixed HB and FB SMs, the proposed topology uses the DC capacitor in the fault breaking circuit to block DC faults, while the FB-MMC only commutates the fault current from the FB-MMC to the fault breaking circuit. Thus, the proposed converter only requires around $10 \%-20 \% \mathrm{FB}$ SMs, leading to reduced capital cost and losses compared to typical hybrid MMC. The optimal ratio of the FB-MMC and $\mathrm{HB}-\mathrm{MMC}$ is assessed and comparative studies show superiority of the proposed topology over other alternatives. A case study with $10 \%$ FB SMs demonstrates the validity of the proposed hybrid MMC for DC fault blocking and post-fault system restart.
\end{abstract}

Index Terms-DC fault, DC circuit breaker (DCCB), HVDC transmission, hybrid multilevel converter, modular multilevel converter (MMC).

\section{INTRODUCTION}

$\mathrm{H}$ igh-voltage DC (HVDC) transmission systems based on modular multilevel converters (MMCs) have developed rapidly due to their significant advantages. However, the behaviors and characteristics of half-bridge (HB) submodule (SM) based MMC (HB-MMC) to DC faults are major issues to be considered in applications. In the event of a DC short circuit, high AC currents flow through the freewheeling diodes of HB$\mathrm{MMC}$ from the $\mathrm{AC}$ to the $\mathrm{DC}$ side, which requires additional measures and could potentially cause serious damage to the converters and associated semiconductor devices [1-3].

Conventional mechanical DC circuit breakers (DCCBs) have low conduction losses. However, their response is too slow and the semiconductors still endure high current stress during the response time. The solid-state DC circuit breaker can achieve fast interruption but at high capital cost and significant on-state operation losses [4]. The hybrid DC circuit breaker combines the advantages of the mechanical and solid-state DCCBs, where a mechanical path serves as main conduction path with minimal losses during normal operation, and a parallel-connected solid-state breaker is used for DC fault isolation $[5,6]$. However, it has relatively large footprint and its capital cost is still very high $[7,8]$.

R. Li and L. Xu are with the Department of Electronic and Electrical Engineering, University of Strathclyde, Glasgow, G1 1XW UK (e-mail: rui.li@strath.ac.uk, lie.xu@strath.ac.uk).

L. Yu is with the School of Electrical and Information Engineering, Tianjin University, Tianjin, 300072, China (e-mail: lujie.yu@ outlook.com).

L. Yao is with the School of Electrical Engineering and Automation, Wuhan University, Wuhan, 430072, China (yaoliangzhong@whu.edu.cn).
In addition to the use of DCCB for fault isolation, different fault blocking converter topologies have been proposed. In the full-bridge (FB) SM based MMC (FB-MMC), the FB SM capacitors can be inserted into the circuit in either polarity. This feature allows the FB-MMC to block DC faults and offer greater controllability [9]. However, FB-MMC doubles the required semiconductors compared to $\mathrm{HB}-\mathrm{MMC}$, leading to additional cost and losses.

In addition to the FB SM, various SM topologies have been proposed, e.g. clamp double (CD) SM [10], cross connected (CC) SM [11, 12], unipolar FB SM [13], unipolar CC SMs [14], etc. These SMs use additional semiconductor switches to change the current path during DC faults and block the fault currents fed from the AC side, while operate in a similar way with HB SM during normal operation. However, the introduction of additional semiconductors leads to higher losses and cost.

The alternate-arm multilevel converter (AAC) presented in [15] can block DC faults with reduced semiconductor losses compared to the FB-MMC. However, the direct switches in the AAC arm require series connection of large numbers of IGBTs [16]. Based on HB-MMC, the hybrid cascaded MMC (HC$\mathrm{MMC}$ ) is proposed in [17], where cascaded FB chains are connected on the AC side of HB-MMC to provide reverse blocking voltage after the converter is blocked following the fault. This topology has lower losses and cost than FB-MMCs but the cascaded FB chains need to be coordinately controlled with the HB-MMC part.

Reference [18] proposes a hybrid MMC (H-MMC), which mixes FB and HB SMs in each arm to obtain DC fault blocking capability with lower losses than the FB-MMC. Also, it can generate negative voltage, which enables greater controllability of the converter $[18,19]$. The ratio between the FB and HB number is typically fix at one to effectively block DC faults. The ratio greater than one is also discussed in [18] to transfer more power than the conventional MMC by utilizing the negative output voltage capability of the FB SMs. However, with more HB SMs replaced by FB SMs, the power losses are increased.

Another hybrid MMC is proposed in [20], where only one HB SM is replaced by a FB SM in the lower arm. During DC faults, all the HB SMs in the upper arm are commanded to output zero voltage to create an artificial AC short circuit so as to bypass the fault currents fed by the AC grid from DC side. Meanwhile, all the SMs in the lower arm are turned off thus the FB SM provides blocking voltage to rapidly reduce the DC current to zero. As a result, the DC switches can be opened at zero current to isolate the DC fault and then all the HB SMs in 
the upper arm can be deactivated. However, before the opening of the DC switches, large fault currents flow through the IGBTs in the upper arm, leading to severe overcurrent.

In order to overcome the above problems, a hybrid MMC with reduced FB SMs (RFB-MMC) is proposed in this paper and its operation including DC fault blocking and restart capabilities is researched. The proposed converter is introduced in section II including the converter topology and typical characteristics. In section III, the operating principle considering DC fault blocking and restart procedure is detailed. Section IV presents the design principles of the proposed topology, considering cost, loss, and control strategy. The performance of the proposed RFB-MMC is assessed in section $\mathrm{V}$ using simulations and section VI concludes the paper.

\section{Proposed Hybrid MMCs With REDUCED FB SMS}

Fig. 1 shows a generic version of the proposed hybrid MMC with reduced FB SMs. Its main power stage consists of a series connection of HB-MMC and FB-MMC on their DC sides. A fault breaking circuit, composed of an ultra-fast disconnector (UFD), a residual DC current breaker (RB), and a DC capacitor $C_{F}$, is connected in parallel with the FB-MMC on the DC side to provide DC fault blocking and DC circuit breaking capability.

For the conventional hybrid MMC, the negative voltage provided by the FB SMs needs to be greater than the peak AC line-to-line voltage to block DC faults. Thus, typically it requires $50 \% \mathrm{FB} \mathrm{SMs}$, leading to higher cost and power losses than HB-MMCs $[18,19]$. In contrast, in the proposed topology, the FB-MMC only commutates the fault current from the FBMMC to the fault breaking circuit and does not need to match the AC side voltage to block DC faults. Thus, the ratio $k$ between the FB SM number $N_{F B}$ and the total SM number $N$ in each arm of the RFB-MMC can be much less than the typical value of $50 \%$, i.e.:

$$
k=\frac{N_{F B}}{N}=\frac{N_{F B}}{N_{F B}+N_{H B}}<50 \%
$$

where $N_{H B}$ is the HB SM number per arm for the HB-MMC. As will be discussed in section IV, $k$ can be reduced from the typical value of $50 \%$ in $\mathrm{H}-\mathrm{MMC}$ to $10 \%$, leading to reduced losses and capital cost.

For modular design, the FB and HB SM capacitors have the same voltage $V_{C}$ :

$$
V_{C}=\frac{V_{d c F B}}{N_{F B}}=\frac{V_{d c H B}}{N_{H B}}=\frac{V_{d c}}{N}=\frac{k V_{d c}}{N_{F B}}
$$

where $V_{d c F B}, V_{d c H B}$ and $V_{d c}$ are the DC voltages of the FB-MMC, HB-MMC and RFB-MMC, respectively. Considering unity modulation index, the peak AC line-to-line voltages $V_{a c F B}$ and $V_{a c H B}$ of the FB-MMC and HB-MMC are assumed as (3) and (4), respectively:

$$
\begin{gathered}
V_{a c F B}=\frac{\sqrt{3}}{2} k V_{d c} \\
V_{a c H B}=\frac{\sqrt{3}}{2}(1-k) V_{d c} .
\end{gathered}
$$

According to Kirchhoff's current law, the DC currents are governed by (5) from Fig. 1:

$$
I_{d c H B}=I_{d c F B}+I_{C F}=I_{R B}
$$

where $I_{d c H B}$ and $I_{d c F B}$ are the DC currents of HB-MMC and FBMMC, respectively, and $I_{C F}$ and $I_{R B}$ are the currents flowing through capacitor $C_{F}$ and residual breaker RB, respectively.

During normal operation, i.e. non-DC fault conditions, the proposed topology operates in the same way as the conventional HB-MMCs including the same AC fault ride-through capability [21].

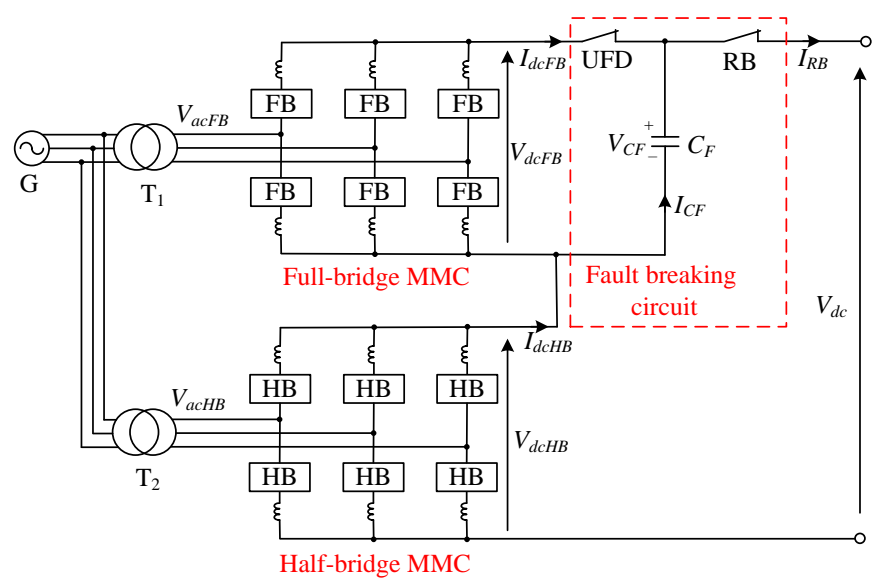

Fig. 1. Proposed hybrid MMC with reduced FB SMs.

\section{OPERATING PRINCIPLE OF THE PROPOSED RFB-MMC}

The operating principles of the proposed scheme, including DC fault blocking and system restart, are presented in this section.

\section{A. DC Fault Blocking}

The principle idea of the proposed topology is to use DC capacitor $C_{F}$ rather than FB SMs to block DC faults such that $k$ ration can be significantly reduced. The operation of the circuit can be explained as follows.

- During normal operation (Stage I), UFD and RB are closed and the capacitor voltage $V_{C F}$ equals to the nominal FBMMC DC voltage while the capacitor current $I_{C F}$ is around zero, as shown in Fig. 2 (a). Both the FB-MMC and HBMMC transmit power during normal operation.

- In the event of DC faults, the HB SM capacitors and the capacitor $C_{F}$ are initially discharged as illustrated in Stage II shown in Fig. 2 (b). The currents $I_{d c H B}$ and $I_{C F}$ thus increase, which can be used to detect the fault.

- Both the HB-MMC and FB-MMC are then blocked after fault detection and the FB-MMC provides negative blocking voltage to its AC side, while also suppresses its DC current $I_{d c F B}$ to zero. Thus, all the DC fault current from the HB-MMC $I_{d c H B}$ is commutated to the capacitor $C_{F}$. As the current flowing through the FB-MMC is zero as expressed by (6), the UFD can be opened under zero current (Stage III, Fig. 2 (c)).

$$
I_{C F}=I_{d c H B}, \quad I_{d c F B}=0
$$

- The DC fault current charges the capacitor $C_{F}$, and once the capacitor voltage $V_{C F}$ is greater than the peak AC line-toline voltage $V_{a c H B}$, as depicted by (7), the uncontrolled fault current from the HB-MMC AC side will be suppressed and 
Electrical and Electronic Engineering Copyright. The copy of record is available at IEEE Xplore Digital Library.

the DC fault blocked (Stage IV, Fig. 2 (d)).

$$
\left|V_{C F}(t)\right|=\left|V_{d c F B}-\frac{1}{C_{F}} \int_{t_{0}}^{t} I_{C F} d t\right| \geq V_{a c H B}
$$

where $t_{0}$ is the instant when the DC fault occurs.

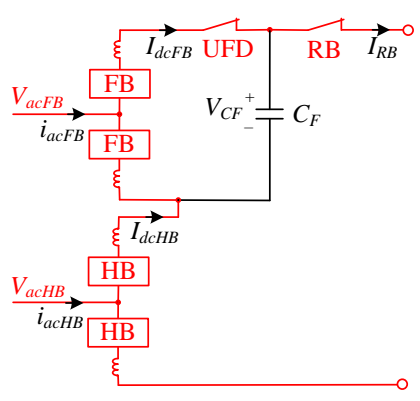

(a)

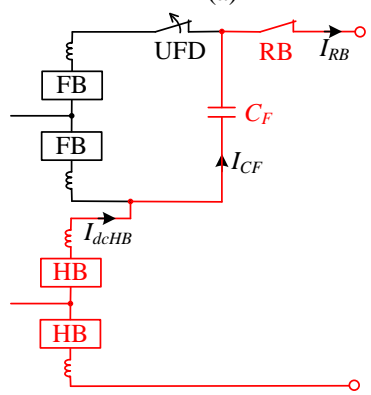

(c)

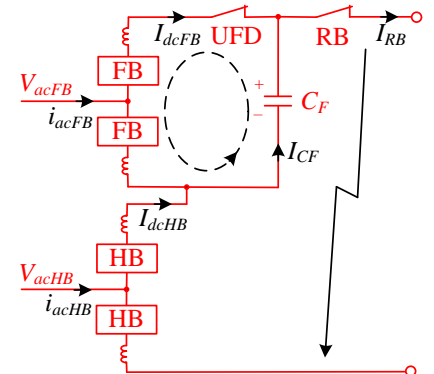

(b)

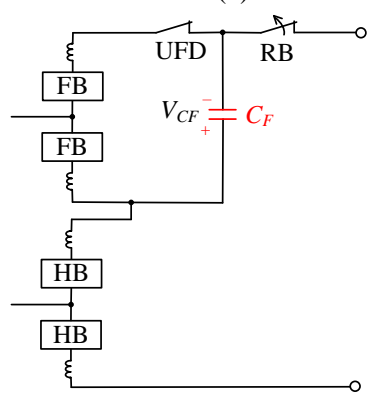

(d)
Fig. 2. Single-phase illustration of fault current commutation of the RFBMMC: (a) Stage I: normal operation, (b) Stage II: fault current commutation, (c) Stage III: capacitor $C_{F}$ reverse charging, and (d) Stage IV: fault blocking.

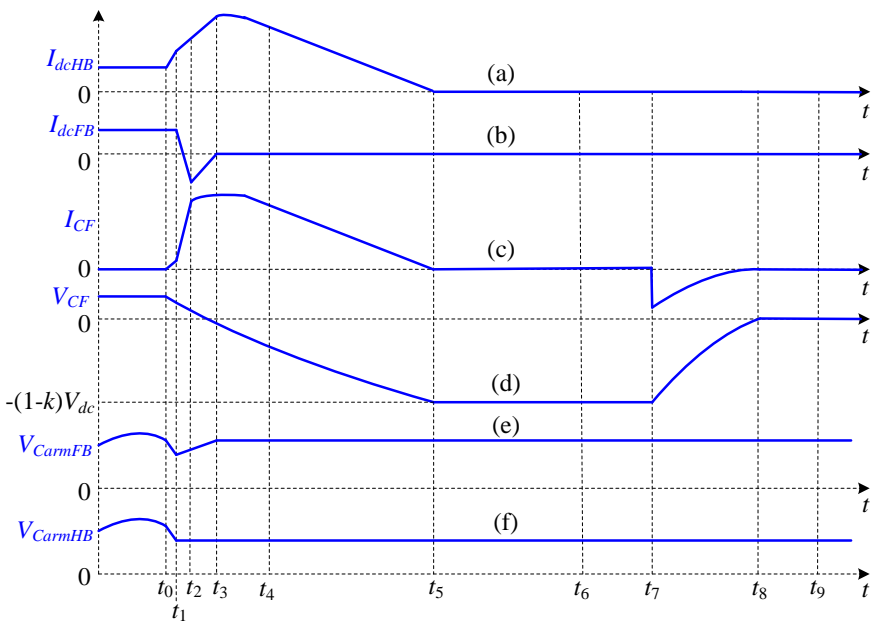

Fig. 3. Illustration of the typical waveforms of the RFB-MMC during faults: (a) HB-MMC DC current, (b) FB-MMC DC current, (c) capacitor $C_{F}$ current, (d) capacitor $C_{F}$ voltage, (e) sum of SM capacitor voltages of FB-MMC, and (f) sum of SM capacitor voltages of HB-MMC.

The typical waveforms of the proposed converter during DC faults are illustrated in Fig. 3, which are described as follows.

- When a DC fault occurs at $t=t_{0}$, capacitor $C_{F}$ discharges and its current $I_{C F}$ increases. Meanwhile, SM capacitors of HB$\mathrm{MMC}$ are also discharged, leading to the increase of the DC current $I_{d c H B}$.

- Once the DC fault is detected at $t=t_{1}$ by either over current detection or other suitable methods [22], both the FB and $\mathrm{HB}$ converters of the RFB-MMC are blocked and the SM capacitors stop discharging.

- After the converters are blocked at $t=t_{1}$, the fault currents flow through the antiparallel diodes in the arms and charge the SM capacitors of the FB-MMC, as shown in Fig. 4 (a), which considers one SM per arm for ease of illustration. Fig. 4 (a) can be equivalent as Fig. 4 (b) and as seen, depending on the arm current directions, the upper and lower arms of the FB-MMC provide positive and negative voltages. Thus, the sum of the upper and lower arm voltages of the FBMMC is zero $\left(V_{d c F B}-V_{d c F B}\right)$ during $t_{1} \sim t_{2}$. Due to the capacitor voltage $V_{C F}$, the DC current $I_{d c F B}$ decreases to zero and reverses until $t=t_{2}$ when one of the upper and lower arm currents in a phase becomes zero. At this instant, the blocking voltage provided by the FB-MMC is increased from zero to $2 V_{d c F B}$ (i.e. $V_{d c F B}+V_{d c F B}$ ), as seen from Fig. 4 (c). The DC current $I_{d c F B}$ then starts to decrease and is suppressed to zero at $t=t_{3}$. The fault current is thus commutated to the capacitor $C_{F}$ and the FB-MMC is bypassed, as illustrated in Fig. 2 (c). Therefore, UFD is opened at zero current at $t=t_{4}$ after certain delays. Typical UFD opening time is $2 \mathrm{~ms}$, and thus, it takes around $2 \mathrm{~ms}$ from fault detection at $t=t_{1}$ to the opening of the UFD at $t=t_{4}$.

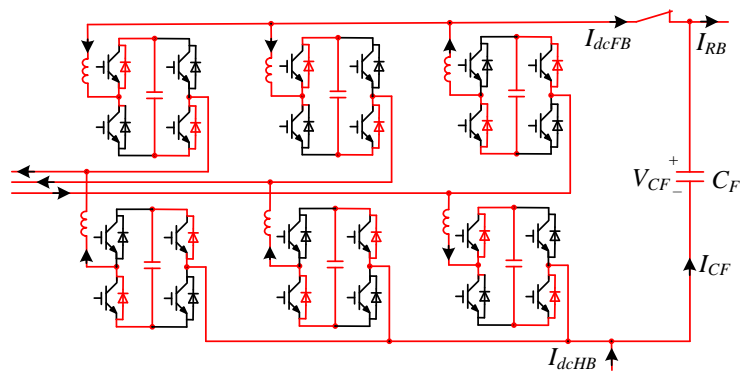

(a)

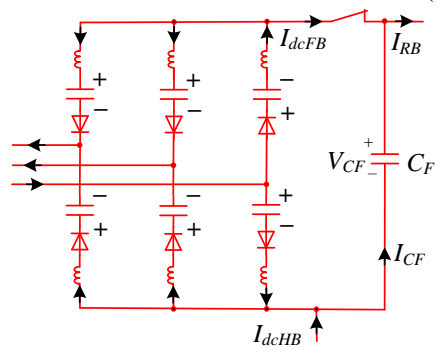

(b)

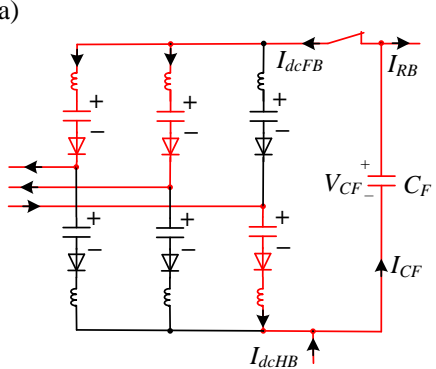

(c)
Fig. 4. Current path illustration during FB-MMC DC current reversal: (a) detailed current path during $t_{1} \sim t_{2}$, (b) equivalent current path during $t_{1} \sim t_{2}$ and (c) equivalent current path during $t_{2} \sim t_{3}$.

- Following the blocking of the RFB-MMC at $t=t_{1}$, the sum of SM capacitor voltages of HB-MMC $V_{C a r m H B}$ remains unchanged seen in Fig. 3 (f), as the fault currents only flow through the antiparallel diodes of the HB SMs and do not charge the HB SM capacitors. In contrast, the FB SMs provide negative voltages to suppress the fault currents, which charge the FB SM capacitors, leading to slight increase of the FB capacitor voltage $V_{C a r m F B}$ from $t=t_{1}$ to $t=t_{3}$ as illustrated in Fig. 3 (e). After the suppression of the FBMMC DC current $I_{d c F B}$ to zero at $t=t_{3}, V_{C a r m F B}$ remains unchanged.

- From fault initiation, fault currents continue being fed to the DC side by the AC grid voltage $V_{a c H B}$ through the 
Electrical and Electronic Engineering Copyright. The copy of record is available at IEEE Xplore Digital Library.

antiparallel diodes of the HB-MMC. The fault current $I_{d c H B}$ flows through $C_{F}$ and its voltage $V_{C F}$ decreases to zero and then reverses. When $V_{C F}$ increases to around $-(1-k) V_{d c}$, the fault current $I_{d c H B}$ reduces to zero at $t=t_{5}$ and the DC fault is thus blocked, as illustrated in Fig. 2 (d), and Fig. 3 (a) and (d). If the residual breaker RB is connected with the faulty DC line, it can be opened at $t=t_{6}$ to physically isolate the RFB-MMC from the faulty DC network. On the other hand, if the DC fault is in other part of the DC network in a multiterminal DC system, i.e. the DC fault is not at the DC line connected to the RFB-MMC, the RB can remain closed.

\section{B. System Restart}

After fault isolation, the RFB-MMCs connected to the healthy part of the DC network need to recover and restart power transmission. This requires $C_{F}$ to be discharged first. In the proposed scheme, a fast discharging resistor $R_{d i s}$ is switched in using a mechanical switch $S_{\text {dis }}$ with the closing time of $20 \mathrm{~ms}$ as shown in Fig. 5. The sequence during restart is detailed as follows.

- After fault isolation (at other part of the DC network so RB remains closed), the mechanical switch $S_{d i s}$ is closed at $t=t_{7}$ and the capacitor $C_{F}$ is discharged as seen in Fig. 3. When the capacitor voltage $V_{C F}$ drops below the peak of the HBMMC AC voltage $V_{a c H B}$ at $t=t_{8}$, AC currents start to flow through the freewheeling diodes and charge the healthy DC cables. Once the DC cables are charged, the charging currents reduce to zero.

- After the capacitor $C_{F}$ is safely discharged, the discharging switch $S_{d i s}$ is opened and the UFD closes at $t=t_{9}$. The FBMMC is thus connected in the circuit and normal operation can be resumed.

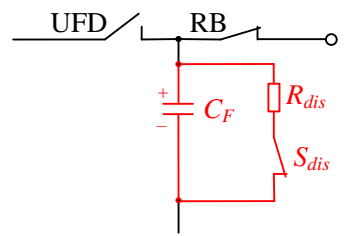

Fig. 5. Discharging circuit for the fault blocking capacitor $C_{F}$ of the RFBMMC.

The fast discharging resistor $R_{\text {dis }}$ only needs to dissipate the energy stored in the capacitor $C_{F}$ during system restart and does not introduce power losses during normal operation, as it is disconnected from the circuit by the mechanical switch $S_{d i s}$.

\section{DESIGN OF THE PROPOSED RFB-MMC}

The design principles of the proposed RFB-MMC are presented in this section, considering cost, losses, and control structures.

\section{A. Cost Consideration}

\section{1) Capacitor $C_{F}$ requirements}

To ensure that UFD is safely opened under zero current at $t=t_{4}$ in Stage III, the reversed DC capacitor voltage $V_{C F}$ needs to be less than the negative voltage $V_{\text {blockFB }}$ provided by the FBMMC, as expressed by (8). Otherwise, $V_{C F}$ will force $I_{d c F B}$ to increase again.

$$
\begin{aligned}
& \left|V_{C F}\left(t_{4}\right)\right|=\left|V_{d c F B}-\frac{1}{C_{F}} \int_{t_{0}}^{t_{4}} I_{C F} d t\right|<V_{\text {blockFB }} . \\
& V_{\text {blockFB }}=2 V_{C} N_{F B}=2 V_{d c F B}=2 k V_{d c}
\end{aligned}
$$

Typical UFD opening time is around $2 \mathrm{~ms}$. Thus, $2 \mathrm{~ms}$ after fault detection and the initiation of the opening of the UFD, the reversed capacitor voltage $V_{C F}$ needs to be less than the blocking voltage $V_{\text {blockFB }}$. Considering the integration of the fault current within the first $2 \mathrm{~ms}$ is largely constant, from (8), the required capacitance $C_{F}$ is inversely proportional to the blocking voltage of the FB-MMC. In the most severe cases tested while considering possible different power flow directions, operation point, etc., the value of the required capacitance $C_{F}$ is $0.5 C_{S M} / N$ in the specific case with $k=20 \%$, where $C_{S M}$ is the SM capacitance. With the variation of $k$, the required $C_{F}$ can be considered as inversely proportional to the ratio $k$ as:

$$
C_{F}=\frac{0.2}{k} \frac{C_{S M}}{2 N}=\frac{C_{S M}}{10 k N} .
$$

The total energy stored in the FB and HB SM capacitors of the RFB-MMC is

$$
\begin{aligned}
E_{S M} & =6 k N \frac{1}{2} C_{S M}\left(\frac{V_{d c}}{N}\right)^{2}+6(1-k) N \frac{1}{2} C_{S M}\left(\frac{V_{d c}}{N}\right)^{2} \\
& =\frac{3 C_{S M}}{N} V_{d c}{ }^{2}
\end{aligned}
$$

From (9) and (10), and assuming the blocking voltage provided by the capacitor $C_{F}$ during DC faults is $(1-k) V_{d c}$, the energy stored in the capacitor $C_{F}$ is expressed as

$$
\begin{aligned}
E_{C F} & =\frac{1}{2} C_{F}\left[(1-k) V_{d c}\right]^{2} \\
& =\frac{C_{S M}}{20 k N}(1-k)^{2} V_{d c}{ }^{2}=(1-k)^{2} \frac{E_{S M}}{60 k}
\end{aligned} .
$$

The FB and HB SMs of the proposed RFB-MMC require the same value of capacitor as that used in the conventional HBMMC and can be in the range of is 30 40 kJ/MVA [23]. From (11), the total required capacitor volume of the RFB-MMC is thus around $1+\frac{(1-k)^{2}}{60 k}$ times that of HB-MMC.

\section{2) Cost evaluation}

The required IGBT number per arm of the proposed RFBMMC is the sum of those in the FB-MMC and HB-MMC:

$$
4 N k+2 N(1-k)=2 N(1+k) \text {. }
$$

Assuming the SM capacitors and semiconductor devices equally share the total cost $C O_{H B-M M C}$ of the conventional HBMMC [24], the semiconductor cost $\mathrm{CO}_{\text {semi }}$ of the proposed $\mathrm{RFB}-\mathrm{MMC}$ is:

$$
C O_{\text {semi }}=\frac{2 N(1+k)}{2 N} \times \frac{C O_{H B-M M C}}{2}=\frac{1+k}{2} C O_{H B-M M C} .
$$

The capacitor cost of the FB and HB SMs in the RFB-MMC $C O_{C S M}$ is the same as that of the conventional HB-MMC:

$$
C O_{C S M}=0.5 C O_{H B-M M C} \text {. }
$$

From (11), the cost of the fault blocking capacitor $C_{F}$ is

$$
C O_{C F}=\frac{E_{C F}}{E_{S M}} \frac{C O_{H B-M M C}}{2}=\frac{(1-k)^{2}}{120 k} C O_{H B-M M C} .
$$


Similar to the residual breaker of hybrid breakers, the residual breaker RB physically isolates the faulty cables after faults are blocked by the RFB-MMC. Thus, its opening speed is not critical and normal mechanical switches can be used. The cost $C O_{\text {else }}$ of the RB, UFD and the discharging circuit is estimated as $0.05 C O_{H B-M M C}[20]$. The total cost of the RFB$\mathrm{MMC}$ is thus calculated as

$$
\begin{aligned}
C O_{R F B} & =C O_{\text {semi }}+C O_{C S M}+C O_{C F}+C O_{S F}+C O_{\text {else }} \\
& =\frac{61 k^{2}+124 k+1}{120 k} C O_{H B-M M C}
\end{aligned}
$$

The total cost of the FB-MMC and the conventional hybrid MMC is estimated as (17) and (18), respectively:

$$
\begin{aligned}
& C O_{F B-M M C}=(1+1) \frac{C O_{H B-M M C}}{2}+\frac{C O_{H B-M M C}}{2}=1.5 C O_{H B-M M C} \\
& C O_{H-M M C}=(1+0.5) \frac{C O_{H B-M M C}}{2}+\frac{C O_{H B-M M C}}{2}=1.25 C O_{H B-M M C} .
\end{aligned}
$$

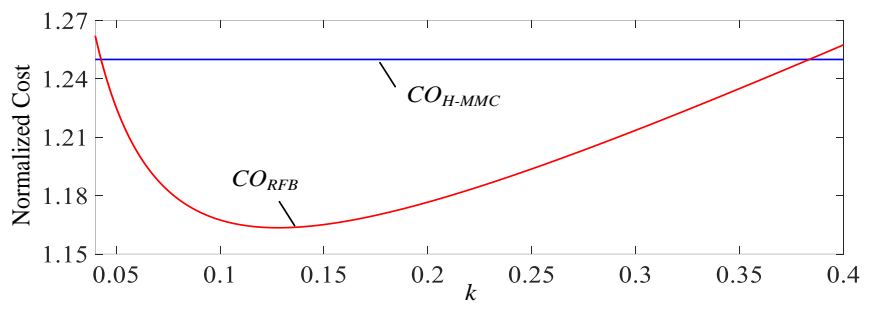

Fig. 6. Comparison of normalized cost between the conventional hybrid MMC and the proposed RFB-MMC with the variation of $k$.

From (16) and (18), the normalized capital costs of the conventional hybrid MMC and the proposed RFB-MMC are plotted in Fig. 6, where the HB-MMC cost $\mathrm{CO}_{H B-M M C}$ is defined as the base value. When $k$ is in the range of $(0.04 \sim 0.38)$, the proposed topology is more cost-effective than the conventional $\mathrm{H}-\mathrm{MMC}$, and the minimum cost $C O_{R F B \min }$ of the RFB-MMC is around $1.16 C O_{H B-M M C}$ from (16), a reduction of $7.2 \%$ compared to the H-MMC cost of $1.25 C O_{H B-M M C}$.

TABLE I

Comparison of the proposed RFB-MMC $(k=0.1)$ with other alternatives.

\begin{tabular}{l|l|l|l|l}
\hline \hline & $\begin{array}{c}\text { HB- } \\
\text { MMC }\end{array}$ & $\begin{array}{c}\text { FB- } \\
\text { MMC }\end{array}$ & $\begin{array}{c}\text { H- } \\
\text { MMC }\end{array}$ & $\begin{array}{c}\text { RFB- } \\
\text { MMC }\end{array}$ \\
\hline $\begin{array}{l}\text { Capacitor stored energy } \\
\text { (kJ/MVA) }\end{array}$ & $30 \sim 40$ & $30 \sim 40$ & $30 \sim 40$ & $35 \sim 47$ \\
\hline No. of semiconductors per arm & $2 N$ & $4 N$ & $3 N$ & $2.2 N$ \\
\hline $\begin{array}{l}\text { No. of semiconductors in } \\
\text { current path per arm }\end{array}$ & $N$ & $2 N$ & $1.5 N$ & $1.1 N$ \\
\hline Cost (pu) & 1 & 1.5 & 1.25 & 1.16 \\
\hline Losses & $1 \%$ & $1.7 \%$ & $1.35 \%$ & $1.07 \%$ \\
\hline DC fault blocking & No & Yes & Yes & Yes \\
\hline \hline
\end{tabular}

According to (12), the required IGBT number per arm of the proposed RFB-MMC with $k=0.1$ is $2.2 N$, a reduction of $26.7 \%$ compared to that of the conventional hybrid MMC ( $3 N)$. From (11), when $k=0.1$, the capacitance requirement of the RFB$\mathrm{MMC}$ is in the range of 35 47 kJ/MVA. The comparison of the proposed RFB-MMC with other alternative MMC configurations is summarized in Table I.

\section{B. Loss Consideration}

The proposed fault breaking circuit is inactive during normal operation. Currents only flow through the mechanical devices UFD and RB, and thus the loss of the fault breaking circuit is negligible.
The conduction loss of the RFB-MMC is proportional to the semiconductor number in the current path per arm, which is expressed as (19) for the proposed RFB-MMC:

$$
2 N_{F B}+N_{H B}=2 k N+(1-k) N=(1+k) N .
$$

Thus, with $k=0.1$, the per arm semiconductor number in the current path of the proposed RFB-MMC is $1.1 N$, lower than that of the conventional hybrid MMC $(1.5 N)$, as listed in Table I. This significantly reduces conduction losses. The switching loss of the RFB-MMC is similar to that of HB-MMC. Assuming the conduction and switching losses of the conventional HBMMC are $0.7 L O_{H B-M M C}$ and $0.3 L O_{H B-M M C}$, respectively [25], the total loss of the proposed RFB-MMC is

$$
\begin{aligned}
& \frac{2 N+2 k N}{2 N} \times 0.7 L O_{H B-M M C}+0.3 L O_{H B-M M C} . \\
& =(1+0.7 \mathrm{k}) L O_{H B-M M C}
\end{aligned}
$$

The losses of the conventional HB-MMC and hybrid MMC are typically $1 \%$ and $1.35 \%$, respectively $[18,25]$. From (20), the loss of the proposed RFB-MMC with $k=0.1$ is calculated as $1.07 \%$, a reduction of $20.7 \%$ compared to the conventional hybrid MMC as listed in Table I.

The capitalized cost associated with losses is typically $3000 € / \mathrm{kW}$ [25] and thus the proposed RFB-MMC further reduces the cost by $10 \mathrm{M} €$ per converter if the conventional hybrid MMC rated at $1200 \mathrm{MW}$ is replaced by the proposed RFB-MMC.

The proposed RFB-MMC shows superiorities over other alternatives in terms of efficiency and required semiconductor number, which are among the major concerns when constructing HVDC projects. The construction, installation and space requirements of various topologies will incur different costs, which need to be taken into consideration in real applications.

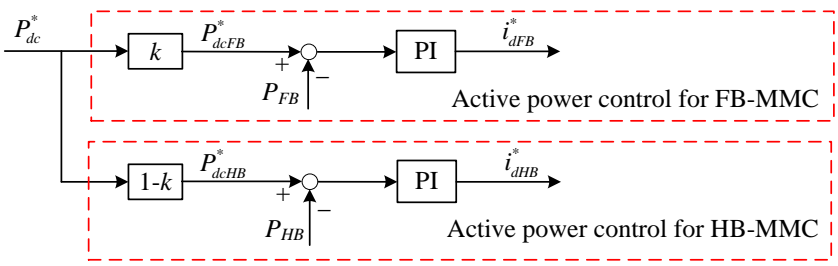

(a)

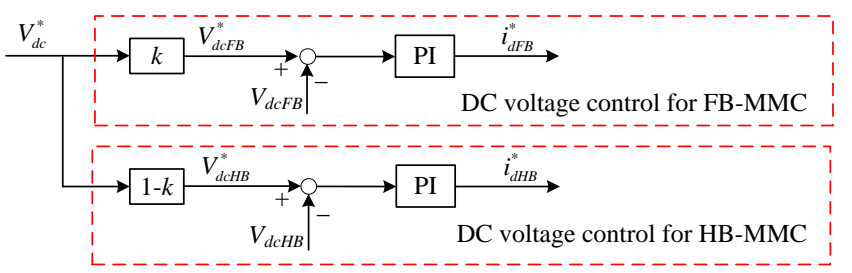

(b)

Fig. 7. Control structure of the proposed RFB-MMC: (a) active power control and (b) DC voltage control.

\section{Control Strategy}

For the proposed topology, the FB-MMC and HB-MMC are connected in series and share the same DC current. To ensure DC voltage sharing according to the ratio $k$ defined by (1), the active power references of the FB-MMC and HB-MMC need to be set as $P_{d c F B}^{*}=k P_{d c}^{*}$ and $P_{d c H B}^{*}=(1-k) P_{d c}^{*}$, respectively, as 
Electrical and Electronic Engineering Copyright. The copy of record is available at IEEE Xplore Digital Library.

illustrated in Fig. 7 (a), where $P_{d c}^{*}$ is the active power reference of the RFB-MMC; $i_{d F B}^{*}$ and $i_{d H B}^{*}$ are the $d$-axis current references of the FB-MMC and HB-MMC, respectively [26].

Similarly, for the RFB-MMC operating on DC voltage control mode, the DC voltage references of the FB-MMC and HB-MMC are set as $V_{d c F B}^{*}=k V_{d c}^{*}$ and $V_{d c H B}^{*}=(1-k) V_{d c}^{*}$, respectively to ensure power sharing and adequate DC voltage control, as illustrated in Fig. 7 (b), where $V_{d c}^{*}$ is the DC voltage reference of the RFB-MMC.

\section{SimULATION}

The DC fault blocking capability of the proposed RFBMMC, and system recovery after fault clearance in a multiterminal system are assessed using Matlab/Simulink simulations. Each arm of the FB-MMC is composed of $32 \mathrm{FB}$ SMs while there are $288 \mathrm{HB}$ SMs in each arm of the HB-MMC. The FB-MMC and HB-MMC of the proposed topology are represented by detailed submodule-based switching function models which consider the gating signal and capacitor voltage ripple of each SM [27]. The $k$ ratio of the FB-MMC is chosen as 0.1 in the studies. Each DC cable is $75 \mathrm{~km}$ long and is modeled as $10 \mathrm{pi}$ sections, while the cable resistance, inductance and capacitance are $20 \mathrm{~m} \Omega / \mathrm{km}, 0.5 \mathrm{mH} / \mathrm{km}$ and 0.27 $\mu \mathrm{F} / \mathrm{km}$, respectively [22].

The blocking capacitor $C_{F}$ is simply modelled as a lumped ideal capacitor while the parasitic inductance and resistance are neglected as they do not have significantly influence on the DC fault blocking performance of the proposed topology. This is because during the respective fault current commutation stage and capacitor $C_{F}$ reverse charging stage shown in Fig. 2 (b) and (c), the much larger arm resistances and inductances of the FBMMC and HB-MMC are always connected in series with the parasitic resistance and inductance of $C_{F}$. Thus, for simplicity, the parasitic inductance and resistance are neglected and an ideal capacitor is used to represent the blocking capacitor $C_{F}$.

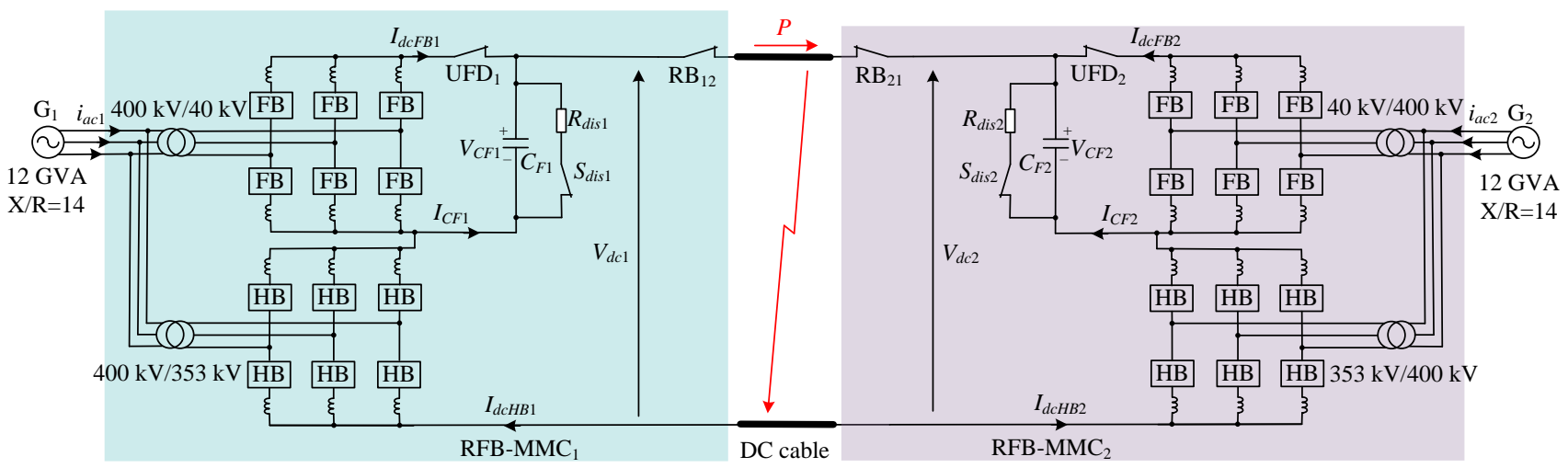

Fig. 8. HVDC link based on the proposed RFB-MMC.

TABLE II

Nominal Parameters of RFB-MMC ${ }_{1}$ and RFB-MMC . $_{2}$

\begin{tabular}{|c|c|c|}
\hline \multicolumn{2}{|r|}{ PARAMETER } & Nominal Value \\
\hline \multirow{7}{*}{ FB-MMC } & DC-link voltage & $80 \mathrm{kV}$ \\
\hline & Power rating & $120 \mathrm{MW}$ \\
\hline & SM number per arm & 32 \\
\hline & SM capacitor voltage & $2.5 \mathrm{kV}$ \\
\hline & SM capacitance $C_{S M}$ & $8 \mathrm{mF}$ \\
\hline & Arm inductance & $0.05 \mathrm{pu}$ \\
\hline & $\begin{array}{l}\text { Grid- and converter-side } \\
\text { voltages of transformer }\end{array}$ & $400 \mathrm{kV} / 40 \mathrm{kV}$ \\
\hline \multirow{7}{*}{ HB-MMC } & DC-link voltage & $720 \mathrm{kV}$ \\
\hline & Power rating & $1080 \mathrm{MW}$ \\
\hline & SM number per arm & 288 \\
\hline & SM capacitor voltage & $2.5 \mathrm{kV}$ \\
\hline & SM capacitance $C_{S M}$ & $8 \mathrm{mF}$ \\
\hline & Arm inductance & $0.05 \mathrm{pu}$ \\
\hline & $\begin{array}{l}\text { Grid- and converter-side } \\
\text { voltages of transformer }\end{array}$ & $400 \mathrm{kV} / 353 \mathrm{kV}$ \\
\hline \multirow{4}{*}{$\begin{array}{l}\text { Fault } \\
\text { breaking } \\
\text { circuit }\end{array}$} & Capacitance $C_{F}$ & $25 \mu \mathrm{F}$ \\
\hline & Voltage rating of $C_{F}$ & $720 \mathrm{kV}$ \\
\hline & Opening time of UFD & $2 \mathrm{~ms}$ \\
\hline & Opening time of RB & $25 \mathrm{~ms}$ \\
\hline
\end{tabular}

\section{A. DC Fault Blocking}

The DC fault blocking capability of the proposed topology is tested using a point-to-point HVDC link rated at $1.2 \mathrm{GW} / 800$ $\mathrm{kV}$ DC, as illustrated in Fig. 8. The tested system parameters are listed in Table II, and RFB-MMC ${ }_{1}$ and RFB-MMC 2 control the active power and DC voltage, respectively. A solid pole-topole DC fault is applied at the middle of the $75 \mathrm{~km}$ DC cables at $t=0.4 \mathrm{~s}$ and the HVDC stations are blocked once the fault is detected if:

- the capacitor current $I_{C F}$ is over $1500 \mathrm{~A}$, or

- the DC fault current $I_{d c H B}$ of the HB-MMC is over $2 \mathrm{pu}$, or

- $\quad I_{C F}$ is over $500 \mathrm{~A}$ and $I_{d c H B}$ is over $1.5 \mathrm{pu}$.

The simulation results are shown in Fig. 9.

After DC fault occurrence, the DC voltage of HVDC link drops to around zero as shown in Fig. 9 (a) and RFB-MMC ${ }_{1}$ is blocked $210 \mu \mathrm{s}$ after fault initiation. As shown in Fig. 9 (b), the FB-MMC DC current $I_{d c F B}$ reverses and is then quickly suppressed to zero by the FB SMs. The currents flowing through the converter arms and the AC side of the FB-MMC are also suppressed to zero, Fig. 9 (f) and (h). Thus, the FB SMs are not subject to additional thermal stress during DC faults. The fault current $I_{d c H B}$ from the HB-MMC AC side thus only flows through $C_{F}$, as displayed in Fig. 9 (c) and (d), and the 
Electrical and Electronic Engineering Copyright. The copy of record is available at IEEE Xplore Digital Library.

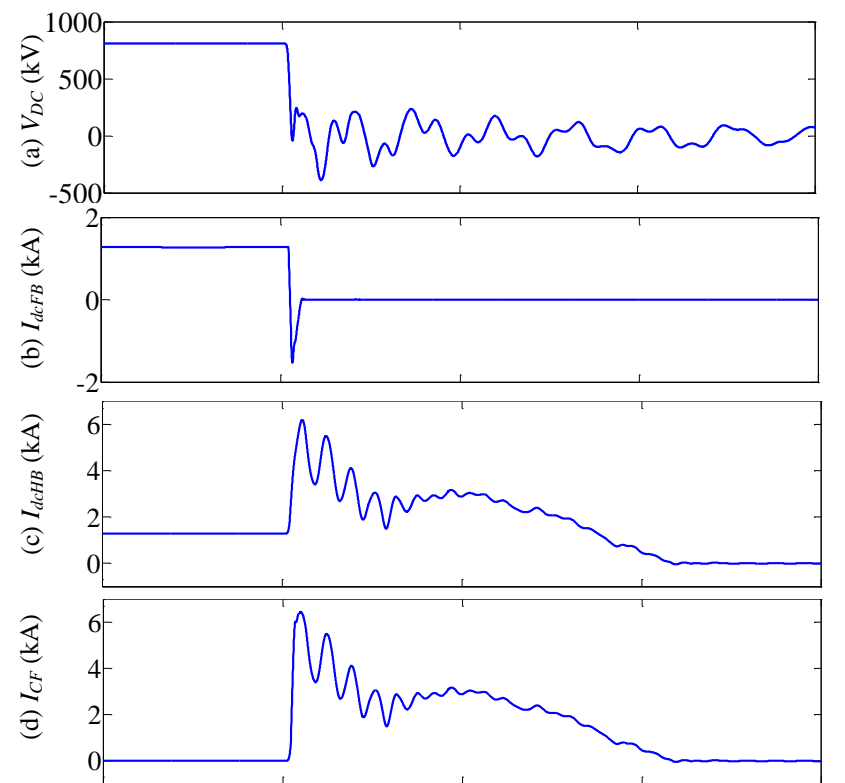

voltage of $C_{F}$ (i.e. $V_{C F}$ ) decreases to zero and then reverses, as shown in Fig. 9 (e). However, $V_{C F}$ is still lower than the FBMMC blocking voltage of $160 \mathrm{kV}$ before $t=0.4022 \mathrm{~s}$ and thus the DC current of the FB-MMC remains at zero, and the UFC is opened under zero current at $t=0.4022 \mathrm{~s}$. The DC fault current continues charging the capacitor $C_{F}$ and, with the increase of the capacitor voltage $V_{C F}$ in opposite to the fault current direction, gradually decreases to zero around $11 \mathrm{~ms}$ after fault detection when $V_{C F}$ reaches around $-720 \mathrm{kV}$, Fig. 9 (c), (d) and (e). The DC fault blocking time of the proposed topology is thus around $11 \mathrm{~ms}$. Similar behaviors can also be observed from the arm and AC currents of the HB-MMC, as seen from Fig. 9 (g) and (i). The peak fault current flowing through the arms of the HB-MMC reaches $3.4 \mathrm{kA}(2.3 \mathrm{pu})$. However, the fault currents only flow through the antiparallel diodes and drop to zero in around $11 \mathrm{~ms}$. The maximum diode $\int i^{2}(t) d t$ is $45 \mathrm{kA}^{2} \mathrm{~s}$ as illustrated in Fig. 9 (k), which is in the safe operation range of the diodes studied [28].
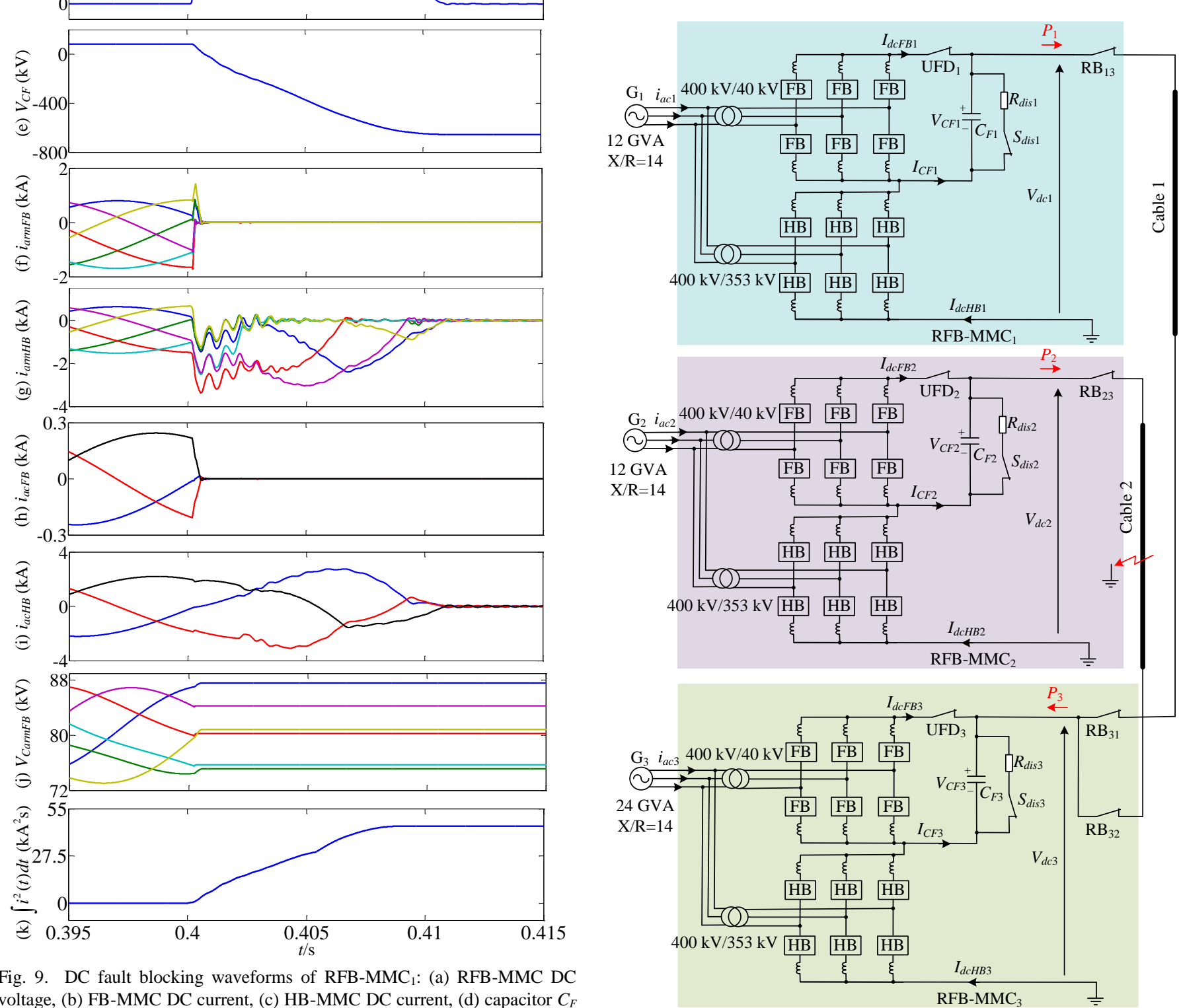

Fig. 9. DC fault blocking waveforms of RFB-MMC 1 : (a) RFB-MMC DC voltage, (b) FB-MMC DC current, (c) HB-MMC DC current, (d) capacitor $C_{F}$ current, (e) capacitor $C_{F}$ voltage, (f) FB-MMC arm currents, (g) HB-MMC arm currents, (h) FB-MMC grid-side AC currents, (i) HB-MMC grid-side AC currents, (j) sum of SM capacitor voltages of FB-MMC, and (k) maximum diode $\int i^{2}(t) d t$ of HB-MMC.

Fig. 10. Three-terminal DC network based on the proposed RFB-MMC.

As shown in Fig. 9 (d), the peak fault current flowing 
Electrical and Electronic Engineering Copyright. The copy of record is available at IEEE Xplore Digital Library.

through the blocking capacitor $C_{F}$ is around $6.5 \mathrm{kA}$ and is much less than those of the DCCB capacitors proposed by Mitsubishi and SCiBreak AB (16 kA and $10 \mathrm{kA}$, respectively) [29, 30].

As the FB SMs of the RFB-MMC only block the fault currents fed from the FB-MMC AC side and commutate the DC current to the fault breaking capacitor $C_{F}$ at the initial stage of the fault, the capacitor voltages of the FB SMs do not have over charging issue, as demonstrated in Fig. 9 (j).

\section{B. DC Fault Ride-through}

The proposed RFB-MMC is also tested using a threeterminal DC network as illustrated in Fig. 10. Stations RFB$\mathrm{MMC}_{1}$ and RFB-MMC 2 operate on power control mode and are connected to the bus-bar of $\mathrm{RFB}-\mathrm{MMC}_{3}$ through residual breakers $\mathrm{RB}_{31}$ and $\mathrm{RB}_{32}$, respectively. RFB-MMC 1 and $\mathrm{RFB}$ $\mathrm{MMC}_{2}$ have the same parameters as the aforementioned pointto-point HVDC link, as listed in Table II. RFB-MMC ${ }_{3}$ controls the DC voltage of the DC network and also has the same parameters as those in Table II except its power rating, SM capacitance $C_{S M}$ and blocking capacitance $C_{F}$ are doubled. A solid pole-to-pole DC fault occurs at the middle of the $75 \mathrm{~km}$ Cable 2 at $t=0.4 \mathrm{~s}$.

After the occurrence of the DC fault, the DC voltage of the network drops to around zero and the three respective stations are blocked $0.3 \mathrm{~ms}, 0.5 \mathrm{~ms}$, and $1 \mathrm{~ms}$ after fault initiation, following the same procedure as detailed in section $\mathrm{V}$ Subsection A. The power transmission is thus interrupted, as shown in Fig. 11 (b) and (c). As observed in Fig. 11 (d) and (e), fault currents flow into the RFB-MMCs at the initial stage of fault occurrence but are quickly suppressed at zero. After fault isolation by opening $\mathrm{RB}_{23}$ and $\mathrm{RB}_{32}$ (fault location is not in the scope of this paper so it considers the fault is located by other methods) at $t=0.421 \mathrm{~s}, \mathrm{RFB}-\mathrm{MMC}_{1}$ and RFB-MMC $\mathrm{C}_{3}$ can be restarted to restore power transmission between them, as detailed as follows.

At $t=0.435 \mathrm{~s}$, the mechanical switch of the discharging circuit $S_{d i s}$ is activated to quickly discharge the capacitor $C_{F}$, as shown in Fig. 11 (i). With the decrease of its voltage $V_{C F}$, currents flow through the HB-MMC and capacitor $C_{F}$ from $\mathrm{AC}$ grid to charge the DC cables, which leads to the increase of the DC voltage and enables soft-restart of the proposed converters, as displayed in Fig. 11 (a), (e), (f), and (h). As shown in Fig. 11 (i), the capacitor voltage $V_{C F}$ decreases to around zero at $t=0.45$ $\mathrm{s}$, and switch $S_{\text {dis }}$ is then opened. UFD is closed at $t=0.452 \mathrm{~s}$ and then the system is now ready to restart.

Station RFB-MMC $\mathrm{C}_{3}$ is firstly enabled $t=0.455 \mathrm{~s}$ to restore the DC voltage as shown in Fig. 11 (a) and RFB-MMC ${ }_{1}$ is activated at $t=0.565 \mathrm{~s}$ and gradually ramps up the active power to the rated value in $50 \mathrm{~ms}$, Fig. 11 (b) and (d). The power transmission between the two healthy stations is thus resumed, Fig. 11 (c) and (e).

With the FB SMs reduced from $50 \%$ to $10 \%$, the proposed RFB-MMCs are still capable of blocking DC faults, avoiding overcurrent of HVDC stations. In a multi-terminal configuration, the healthy parts of the DC network can quickly resume normal operation after fault isolation.

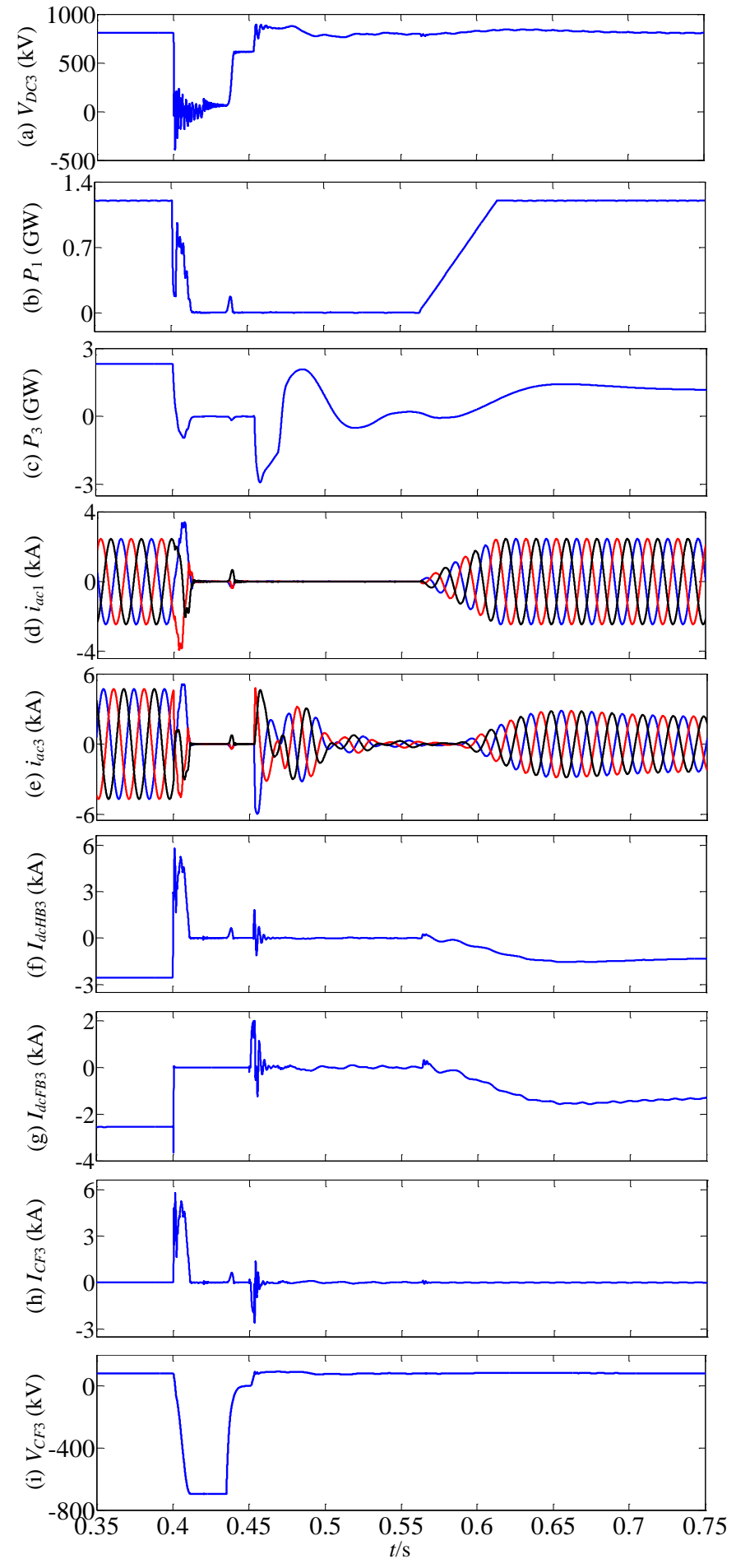

Fig. 11. System restart waveforms during DC faults: (a) RFB-MMC 3 DC voltage, (b) RFB-MMC ${ }_{1}$ active power, (c) RFB-MMC 3 active power, (d) RFB$M_{1} C_{1}$ grid-side AC currents, (e) RFB-MMC 3 grid-side AC currents, (f) HB$\mathrm{MMC}_{3}$ DC current, (g) FB-MMC 3 DC current, (h) capacitor $C_{F 3}$ current, and (i) capacitor $C_{F 3}$ voltage.

\section{CONCLUSION}

This paper proposes a novel hybrid MMC, where a high voltage rating $\mathrm{HB}-\mathrm{MMC}$ is connected in series with a circuit formed by parallel-connected low voltage rating FB-MMC and a fault breaking circuit on DC side. During normal operation, both the FB-MMC and HB-MMC transmit power with the fault 
Electrical and Electronic Engineering Copyright. The copy of record is available at IEEE Xplore Digital Library.

breaking circuit bypassed. During DC faults, the DC capacitor of the fault breaking circuit is charged by the fault currents and provides negative voltage to block faults. The proposed topology significantly reduces the required FB SM number from $50 \%$ for a typical hybrid MMC to $10 \%$, leading lower capital cost and power loss compared to hybrid MMC with 50\% of FB SMs. The DC fault blocking and restart capability, high efficiency, and low capital cost of the proposed RFB-MMC make it attractive for application in HVDC systems.

\section{REFERENCES}

[1] M. B. Ghat and A. Shukla, "A New H-Bridge Hybrid Modular Converter (HBHMC) for HVDC Application: Operating Modes, Control, and Voltage Balancing," IEEE Transactions on Power Electronics, vol. 33, pp. 65376554, 2018.

[2] T. H. Nguyen, K. H. A. Hosani, and M. E. Moursi, "Alternating Submodule Configuration Based MMCs with Carrier-Phase-Shift Modulation in HVDC Systems for DC-Fault Ride-Through Capability," IEEE Transactions on Industrial Informatics, pp. 1-1, 2019.

[3] X. Chen, L. Wang, H. Sun, and Y. Chen, "Fuzzy Logic Based Adaptive Droop Control in Multiterminal HVDC for Wind Power Integration," IEEE Transactions on Energy Conversion, vol. 32, pp. 1200-1208, 2017.

[4] L. L. Qi, A. Antoniazzi, L. Raciti, and D. Leoni, "Design of Solid-State Circuit Breaker-Based Protection for DC Shipboard Power Systems," IEEE Journal of Emerging and Selected Topics in Power Electronics, vol. 5, pp. 260-268, 2017.

[5] N. Ahmed, L. Ängquist, S. Mahmood, A. Antonopoulos, L. Harnefors, S. Norrga, et al., "Efficient Modeling of an MMC-Based Multiterminal DC System Employing Hybrid HVDC Breakers," IEEE Transactions on Power Delivery, vol. 30, pp. 1792-1801, 2015.

[6] O. Cwikowski, J. Sau-Bassols, B. Chang, E. Prieto-Araujo, M. Barnes, O. Gomis-Bellmunt, et al., "Integrated HVDC Circuit Breakers With Current Flow Control Capability," IEEE Transactions on Power Delivery, vol. 33, pp. 371-380, 2018.

[7] E. Kontos, T. Schultz, L. Mackay, L. M. Ramirez-Elizondo, C. M. Franck, and P. Bauer, "Multiline Breaker for HVdc Applications," IEEE Transactions on Power Delivery, vol. 33, pp. 1469-1478, 2018.

[8] A. Mokhberdoran, O. Gomis-Bellmunt, N. Silva, and A. Carvalho, "Current Flow Controlling Hybrid DC Circuit Breaker," IEEE Transactions on Power Electronics, vol. 33, pp. 1323-1334, 2018.

[9] J. Hu, K. Xu, L. Lin, and R. Zeng, "Analysis and Enhanced Control of Hybrid-MMC-Based HVDC Systems During Asymmetrical DC Voltage Faults," IEEE Transactions on Power Delivery, vol. 32, pp. 1394-1403, 2017.

[10] G. Liu, F. Xu, Z. Xu, Z. Zhang, and G. Tang, "Assembly HVDC Breaker for HVDC Grids With Modular Multilevel Converters," IEEE Transactions on Power Electronics, vol. 32, pp. 931-941, 2017.

[11]S. Debnath, Q. Jiangchao, B. Bahrani, M. Saeedifard, and P. Barbosa, "Operation, Control, and Applications of the Modular Multilevel Converter: A Review," Power Electronics, IEEE Transactions on, vol. 30, pp. 37-53, 2015.

[12]A. Nami, L. Jiaqi, F. Dijkhuizen, and G. D. Demetriades, "Modular Multilevel Converters for HVDC Applications: Review on Converter Cells and Functionalities," Power Electronics, IEEE Transactions on, vol. 30, pp. 18-36, 2015.

[13]R. Li, J. E. Fletcher, L. Xu, D. Holliday, and B. W. Williams, "A Hybrid Modular Multilevel Converter With Novel Three-Level Cells for DC Fault Blocking Capability," Power Delivery, IEEE Transactions on, vol. 30, pp. 2017-2026, 2015.
[14]J. Qin, M. Saeedifard, A. Rockhill, and R. Zhou, "Hybrid Design of Modular Multilevel Converters for HVDC Systems Based on Various Submodule Circuits," IEEE Transactions on Power Delivery, vol. 30, pp. 385-394, 2015.

[15]M. M. C. Merlin, T. C. Green, P. D. Mitcheson, D. R. Trainer, R. Critchley, W. Crookes, et al., "The Alternate Arm Converter: A New Hybrid Multilevel Converter With DC-Fault Blocking Capability," Power Delivery, IEEE Transactions on, vol. 29, pp. 310-317, 2014.

[16]M. M. C. Merlin, D. Soto-Sanchez, P. D. Judge, G. Chaffey, P. Clemow, T. C. Green, et al., "The Extended Overlap Alternate Arm Converter: A Voltage-Source Converter With DC Fault Ride-Through Capability and a Compact Design," IEEE Transactions on Power Electronics, vol. 33, pp. 3898-3910, 2018.

[17]R. Li, G. P. Adam, D. Holliday, J. E. Fletcher, and B. W. Williams, "Hybrid Cascaded Modular Multilevel Converter With DC Fault Ride-Through Capability for the HVDC Transmission System," Power Delivery, IEEE Transactions on, vol. 30, pp. 1853-1862, 2015.

[18]R. Zeng, L. Xu, L. Yao, and B. W. Williams, "Design and Operation of a Hybrid Modular Multilevel Converter," Power Electronics, IEEE Transactions on, vol. 30, pp. 1137-1146, 2015.

[19] J. Lee, J. Jung, and S. S. AE, "Balancing of Submodule Capacitor Voltage of Hybrid Modular Multilevel Converter (MMC) under DC Bus Voltage Variation of HVDC System," IEEE Transactions on Power Electronics, pp. 1-1, 2019

[20]X. Huang, L. Qi, and J. Pan, "A New Protection Scheme for MMC-based MVDC Distribution Systems with Complete Converter Fault Current Handling Capability," IEEE Transactions on Industry Applications, pp. 1$1,2019$.

[21]L. Shi, G. P. Adam, R. Li, and L. Xu, "Control of Offshore MMC during Asymmetric Offshore AC Faults for Wind Power Transmission," IEEE Journal of Emerging and Selected Topics in Power Electronics, pp. 1-1, 2019.

[22]R. Li, L. Xu, and L. Yao, "DC Fault Detection and Location in Meshed Multiterminal HVDC Systems Based on DC Reactor Voltage Change Rate," IEEE Transactions on Power Delivery, vol. 32, pp. 1516-1526, 2017.

[23]J. Hu, M. Xiang, L. Lin, M. Lu, J. Zhu, and Z. He, "Improved Design and Control of FBSM MMC With Boosted AC Voltage and Reduced DC Capacitance," IEEE Transactions on Industrial Electronics, vol. 65, pp. 1919-1930, 2018.

[24]Q. Song, R. Zeng, Z. Yu, W. Liu, Y. Huang, W. Yang, et al., "A Modular Multilevel Converter Integrated With DC Circuit Breaker," IEEE Transactions on Power Delivery, vol. 33, pp. 2502-2512, 2018.

[25]T. Jonsson, P. Lundberg, S. Maiti, and Y. Jiang-Häfner, "Converter technologies and functional requirements for reliable and economical HVDC grid design," Cigre Canada, Calgary, Canada, 2013.

[26]X. Chen, L. Yan, X. Zhou, and H. Sun, "A Novel DVR-ESS-Embedded Wind-Energy Conversion System," IEEE Transactions on Sustainable Energy, vol. 9, pp. 1265-1274, 2018.

[27]R. Li, L. Xu, and D. Guo, "Accelerated switching function model of hybrid MMCs for HVDC system simulation," IET Power Electronics, vol. 10, pp. 2199-2207, 2017.

[28] Infineon Technologies AG, FZ1200R45HL3 datasheet, Feb 2018.

[29] K. Tahata, S. Ka, S. Tokoyoda, K. Kamei, K. Kikuchi, D. Yoshida, et al., "HVDC circuit breakers for HVDC grid applications," in Proc. Cigré AORC Technical Meeting, Tokyo, Japan, 2014.

[30]L. Ängquist, A. Baudoin, S. Norrga, S. Nee, and T. Modeer, "Low-cost ultra-fast DC circuit-breaker: Power electronics integrated with mechanical switchgear," in 2018 IEEE International Conference on Industrial Technology (ICIT), 2018, pp. 1708-1713. 


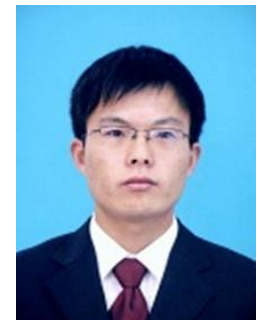

Rui Li received the M.S. and Ph.D degrees in electrical engineering from Harbin Institute of Technology, Harbin, China, in 2008 and 2013, respectively. He is a researcher with University of Strathclyde in Glasgow, UK, since 2013.

His research interests include HVDC transmision system, grid integration of renewable power, power electronic converters, and energy conversion.

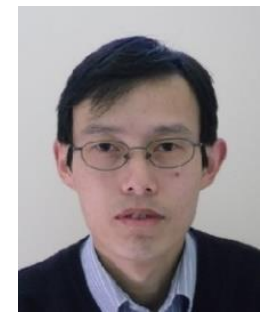

Lie Xu (M'03-SM'06) received the B.Sc. degree in Mechatronics from Zhejiang University, Hangzhou, China, in 1993, and the Ph.D. degree in Electrical Engineering from the University of Sheffield, Sheffield, UK, in 2000.

$\mathrm{He}$ is currently a Professor at the Department of Electronic \& Electrical Engineering, University of Strathclyde, Glasgow, UK. He previously worked in Queen's University of Belfast and ALSTOM T\&D, Stafford, UK. His research interests include power electronics, wind energy generation and grid integration, and application of power electronics to power systems. He is an Editor of IEEE Transactions on Power Delivery and IEEE Transactions on Energy Conversion.

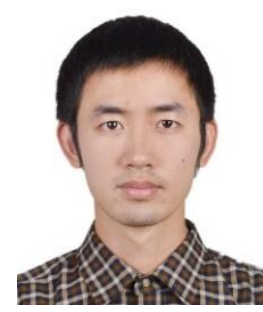

Lujie Yu received the B.S. degree from North China Electric Power University (NCEPU), Baoding, China, in 2012, M.S. degree from NCEPU, Beijing, China, in 2015, Ph.D degree in Electronic \& Electrical Engineering, University of Strathclyde, Glasgow, UK in 2019. Currently, he is a lecturer with School of Electrical and Information Engineering, Tianjin University, Tianjin, China.

His research interests include HVDC transmision system and wind power integration.

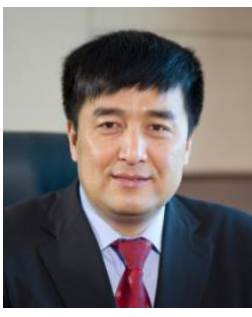

Liangzhong Yao (SM'12) is the Professor in the School of Electrical Engineering and Automation at Wuhan University, Hubei, China. He received the M.Sc. and Ph.D. degrees in electrical power engineering from Tsinghua University, Beijing, China, in 1989 and 1993, respectively.

He was a Postdoctoral Research Associate at the University of Manchester (formerly the University of Manchester Institute of Science and Technology), Manchester, U.K., from 1995 to 1999; a Senior Power System Analyst in the Network Consulting Department at ABB U.K. Ltd. from 1999 to 2004; the Department Manager for Network Solutions, Renewables and Smart Grids Technologies at ALSTOM Grid Research \& Technology Centre, Stafford, U.K., from 2004 to 2011; the Vice President at State Grid Electric Power Research Institute (i.e. NARI Group) in Nanjing from 2011-2012, and the Vice President and Honorary Chief Engineer at China Electric Power Research Institute (CEPRI) in Beijing, from 2011 to 2018.

Dr. Yao is a Chartered Engineer, a Fellow of the IET, and a member of CIGRE. 